
ambiente protegido. Horticultura Brasileira 25: 327-332.

\title{
Critérios para a determinação da dose de nitrogênio a ser aplicada no tomateiro em ambiente protegido
}

\author{
Charles de Araujo ${ }^{1}$; Paulo Cezar Rezende Fontes²; Carlos Sigueyuki Sediyama²; Maurício Bernardes Coelho² \\ ${ }^{1}$ CEFET Cuiabá, 78106-970-Cuiabá-MT; ${ }^{2}$ UFV, Depto. Fitotecnia, 36570-000 Viçosa-MG (bolsistas CNPq); charledearaujo@yahoo.com.br, \\ pacerefo@ufv.br
}

\begin{abstract}
RESUMO
Avaliou-se o efeito de critérios baseados na análise do teor de nitrato no solo, na produtividade esperada de frutos e em resultados de experimentos anteriores para a recomendação da dose de fertilizante nitrogenado a ser aplicada no tomateiro em ambiente protegido. Dois experimentos foram conduzidos em casa de vegetação, sendo um em solo previamente lixiviado com água (Experimento 1) e outro sem lixiviação (Experimento 2), a fim de simular condições de alto e baixo teor inicial de nitrato no solo. Seis tratamentos foram avaliados no delineamento de blocos ao acaso, com quatro repetições. Os tratamentos foram baseados nos critérios: 1) produtividade esperada de frutos e contribuições do solo e do fertilizante (PESF); 2) quantidade esperada de $N$ no fruto e contribuição do solo (QECS); 3) quantidade esperada de $\mathrm{N}$ no fruto (QEFR); 4) dose recomendada experimentalmente de $280 \mathrm{~kg} \mathrm{ha}^{-1}$ de $\mathrm{N}$, aplicada parceladamente via gotejamento, em cobertura a cada 14 dias (DRCO); 5) mesma dose utilizada no tratamento anterior, mas aplicada em dose total no momento do transplante (DRTR); 6) não aplicação de fertilizante nitrogenado (TEST). As doses variaram de 0 a 570,8 $\mathrm{kg} \mathrm{ha}^{-1}$ de $\mathrm{N}$, dependendo do tratamento. Em ambos os experimentos as produções total e comercial de frutos não diferiram significativamente entre os tratamentos que receberam N. Maiores produções foram obtidas pela utilização do critério PESF. De forma geral, as eficiências de uso, de utilização e agronômica do $\mathrm{N}$ diminuíram à medida que a quantidade de $\mathrm{N}$ aplicada aumentou nos dois experimentos. Os critérios PESF e DRTR proporcionaram maior produtividade e eficiência na recuperação do $\mathrm{N}$ derivado do fertilizante.
\end{abstract}

Palavras-chave: Lycopersicon esculentum, adubação nitrogenada, nitrato no solo, produção esperada de frutos, fertirrigação, eficiência de uso do nitrogênio.

\begin{abstract}
Criterions to rate nitrogen determination to be applied in the tomato plant in unheated greenhouse

We evaluated the effect of criterions based on soil nitrate level, the expected fruit yield and previous experiments results to recommend nitrogen fertilizer rate to be applied in the tomato plants in unheated greenhouse. Two experiments were carried out, one in soil previously leached with water (Experiment 1) and the other without leaching (Experiment 2), aiming to simulate high and low soil nitrate level conditions. Six treatments were evaluated in a randomized complete-block design with four replicates. The treatments were based on the criterions: 1) fruit expected yield and soil and fertilizer contributions (PESF); 2) expected $\mathrm{N}$ amount in fruit and soil contribution (QECS); 3) expected $\mathrm{N}$ amount in fruit (QENF); 4) $280 \mathrm{~kg} \mathrm{ha}^{-1}$ of $\mathrm{N}$, the experimental recommended rate drip applied every 14 days (DRCO); 5) same as the prior treatment, but all $\mathrm{N}$ furrow applied at the transplant time (DRTR); 6) without nitrogen fertilizer application (TEST). The $\mathrm{N}$ rates varied from 0 to $570.8 \mathrm{~kg} \mathrm{ha}^{-1}$. In both experiments the total and marketable fruits yield did not differ significantly among treatments which received $\mathrm{N}$. Higher yields were obtained using the PESF criterion. In a general way, the $\mathrm{N}$ use, utilization and agronomic efficiency decreased as $\mathrm{N}$ applied amount increased in the two experiments. The PESF and DRTR criterions provided higher tomato yield and $\mathrm{N}$ fertilizer recovery efficiency.
\end{abstract}

Keywords: Lycopersicon esculentum, nitrogen fertilizer manage, soil nitrate, expected yield of fruits, fertigation, nitrogen use efficiency.

\section{(Recebido para publicação em 14 de março de 2006; aceito em 11 de julho de 2007)}

A otimização da recomendação da fertilização nitrogenada é necessária para obtenção de alta produtividade e máximo retorno econômico do tomateiro. Entretanto, os processos de nitrificação, lixiviação, volatilização e desnitrificação afetam a disponibilidade de nitrogênio (N) no solo, tornando a recomendação da adubação nitrogenada difícil de ser quantificada. Além disso, fatores como irrigação, regime pluviométrico, modo de aplicação do fertilizante, quantidade de matéria orgânica do solo, cultura antecessora, conteúdo original de $\mathrm{N}$ no solo, tipo de solo e potencial de produção da cultura no específico sistema de produção utilizado também dificultam a quantificação correta da dose de $\mathrm{N}$ a ser aplicada.

No Brasil, a adubação nitrogenada do tomateiro é recomendada de maneira empírica, baseando-se na experiência do produtor ou, raramente, em relações derivadas de doses recomendadas e produtividades da cultura. Neste caso, é utilizada uma dose fixa, sem a preocupação com a otimização econômica, com o aumento na eficiência de uso e de recuperação do fertilizante nitrogenado, com a quantidade de $\mathrm{N}$ residual deixada no solo por ocasião da colheita e com a produtividade da cultura. Conseqüentemente, ao longo do tempo esse empirismo pode resultar em maior quantidade de $\mathrm{N}$ mineral no solo e no aumento do risco de lixiviação do nitrato $\left(\mathrm{N}-\mathrm{NO}_{3}\right)$.

$\mathrm{O}$ acúmulo excessivo de $\mathrm{N}$ no solo acompanhado do aumento de lixiviação do $\mathrm{N}-\mathrm{NO}_{3}$ pode ser evitado se a determinação da dose de $\mathrm{N}$ for realizada com base em sua disponibilidade no solo antes do plantio, essencialmente do $\mathrm{N}$ $\mathrm{NO}_{3}$ em solos sem aplicação de resíduo orgânico (Schröder et al., 2000). Este critério, denominado de método do $\mathrm{N}$ mineral $\left(\mathrm{N}_{\min }\right)$, é adotado em alguns paí- 
ses para várias culturas (Neeteson, 1995). A interpretação do método $\mathrm{N}_{\min }$ baseia-se numa relação linear do conteúdo de N-mineral no solo e a dose ótima do fertilizante nitrogenado (Wadman et al., 1990; Warncke, 1996). Essa relação é obtida em experimentos com múltiplas doses de N (Neeteson, 1995). Alternativamente, a interpretação pode ser feita por meio de nível crítico, acima do qual haverá pequena probabilidade de resposta produtiva em relação à aplicação do N (Heckman, 2002).

A combinação da capacidade de fornecimento de $\mathrm{N}$ pelo solo e pelo fertilizante talvez possa ser apropriada para recomendar a dose de N. A determinação da capacidade do solo pode ser obtida pela divisão da conhecida produção de frutos pelo teor de $\mathrm{N}-\mathrm{NO}_{3}$ no solo no início da instalação da cultura. A capacidade de fornecimento de $\mathrm{N}$ pelo fertilizante é obtida pela divisão da diferença entre as produções obtidas com a dose ótima de $\mathrm{N}$ aplicada e com a ausência de aplicação de $\mathrm{N}$ pela dose ótima de $\mathrm{N}$ aplicada. A combinação pressupõe a existência de dados de experimentos conduzidos anteriormente mas em condições de cultivo semelhantes.

Em regiões úmidas ou que receberam grande quantidade de material orgânico, o comportamento do N no solo é mais complexo e dinâmico e tem havido dificuldades em se utilizar apenas a análise do teor de $\mathrm{N}$-total ou $\mathrm{N}-\mathrm{NO}_{3}$ no solo. Nesse caso, a determinação da quantidade de $\mathrm{N}$ a ser aplicada pode ser baseada no potencial de produção de frutos do sistema (Fontes et al., 2004) ou na necessidade da cultura. Para o tomateiro em ambiente protegido, utilizando os dados de Fayad (1998) pode ser observado que para a produção de 1 t de frutos houve a mobilização de 1,83 kg de N. Esse valor multiplicado pela produção esperada de frutos pode indicar a dose de $\mathrm{N}$.

São raros os trabalhos procurando determinar o potencial do solo e do fertilizante em fornecer $\mathrm{N}$ às plantas, utilizando a determinação do teor de $\mathrm{N}-\mathrm{NO}_{3}$ no solo, a produção esperada de frutos ou a combinação de ambos.

O objetivo deste trabalho foi desenvolver e avaliar critérios, baseados na análise do $\mathrm{N}-\mathrm{NO}_{3}$ no solo, na produtivi- dade esperada de frutos e em resultados de experimentos anteriores, para a recomendação da dose de $\mathrm{N}$ a ser aplicada no tomateiro em ambiente protegido.

\section{MATERIAL E MÉTODOS}

Dois experimentos foram conduzidos simultaneamente em ambiente protegido, na Horta de Pesquisa da UFV, de 09/2002 a 01/2003. Um foi conduzido em Argissolo Vermelho-Amarelo previamente lixiviado, através da aplicação constante de água até o encharcamento do solo, durante 30 dias consecutivos (Experimento 1); outro foi conduzido no mesmo ambiente protegido, mas com os tratamentos sendo aplicados pela segunda vez na mesma parcela, sem lixiviação (Experimento 2). Essa prática teve como objetivo simular condições de alto e baixo teor inicial de nitrato no solo. As características da amostra do solo lixiviado foram: $\mathrm{pH}$ em água $=5,95 ; \mathrm{N}^{-\mathrm{NO}_{3}}$ utilizando-se o extrator $\mathrm{KCl} 1 \mathrm{~mol} \mathrm{~L}^{-1}=123 \mathrm{~kg} \mathrm{ha}^{-1}$; matéria orgânica (carbono orgânico $\mathrm{x}$ 1,724) = 1,79 dag kg-1; P = $44 \mathrm{mg} \mathrm{dm}^{-3}$; $\mathrm{K}=68 \mathrm{mg} \mathrm{dm}^{-3} ; \mathrm{Ca}^{+2}=3,46 \mathrm{cmol} \mathrm{dm}^{-3}$; $\mathrm{Mg}^{+2} 0,85 \mathrm{cmol}_{\mathrm{c}} \mathrm{dm}^{-3} ; \mathrm{H}+\mathrm{Al}=2,60$ $\mathrm{cmol}_{\mathrm{c}} \mathrm{dm}^{-3}$; soma de bases $=4,48 \mathrm{cmol}_{\mathrm{c}}$ $\mathrm{dm}^{-3}$; CTC a pH 7,0 = 7,08 $\mathrm{cmol}_{\mathrm{c}} \mathrm{dm}^{-3}$; saturação de bases $=63 \%$.

Em ambos os experimentos foram avaliados seis tratamentos ou critérios para o manejo da adubação nitrogenada:

Tratamento 1, denominado de PESF, baseado na produtividade esperada de frutos e contribuição do solo e do fertilizante. A dose de nitrogênio $\left(D_{N}\right)$ foi calculada por:

$$
\mathrm{D}_{\mathrm{N}}=\left(\mathrm{C}_{\mathrm{f}} \times \mathrm{P}\right)-\left(1,5 \times \mathrm{NO}_{3}\right) .
$$

Tratamento 2, denominado de QECS, baseado na quantidade esperada de $\mathrm{N}$ no fruto + a contribuição do solo. $\mathrm{D}_{\mathrm{N}}$ foi calculada por:

$$
\mathrm{D}_{N}=\frac{\left.(\mathrm{Q} \times \mathrm{P})-\left(\mathrm{NO}_{3}-\mathrm{N}_{\mathrm{C}}\right)\right\} \times 100}{\mathrm{E}} \text {. }
$$

Tratamento 3, denominado de QENF, baseado na quantidade esperada de $\mathrm{N}$ no fruto. $\mathrm{D}_{\mathrm{N}}$ foi calculada por:

$$
D_{N}=\frac{Q \times P \times 100}{E} \text {. }
$$

Tratamento 4, denominado de DRCO, $280 \mathrm{~kg} \mathrm{ha}^{-1}$ de N, dose recomendada e aplicada totalmente em cobertura.

Tratamento 5, denominado de DRTR, $280 \mathrm{~kg} \mathrm{ha}^{-1}$ de N, dose recomendada e aplicada totalmente no momento do transplante das mudas.

Tratamento 6 ou testemunha, sem aplicação de nitrogênio.

Nas fórmulas acima, $\mathrm{D}_{\mathrm{N}}$ é a dose de $\mathrm{N}$ a ser aplicada $\left(\mathrm{kg} \mathrm{ha}^{-1}\right) ; \mathrm{C}_{\mathrm{f}}$ representa a capacidade de fornecimento de $\mathrm{N}$ pelo fertilizante para a produção de $1 \mathrm{t}$ de frutos (6,03 kg de $\mathrm{N} \mathrm{t}$ fruto $\left.^{-1}\right)$; P é a produção total esperada de frutos (120 t ha-1); 1,5 representa o fator de correção da capacidade do solo em fornecer $\mathrm{N}-\mathrm{NO}_{3}$; Q é a quantidade necessária de $\mathrm{N}$ para produção de $1 \mathrm{t}$ de frutos $(1,83 \mathrm{~kg} \mathrm{~N} \mathrm{t}$ frutos $^{-1}$ ), derivado dos resultados de Fayad (1998); $\mathrm{NO}_{3}$ é a quantidade de $\mathrm{N}-\mathrm{NO}_{3}$ na camada de $0-20 \mathrm{~cm}$ do solo $\left(123,2\right.$ e $101,9 \mathrm{~kg} \mathrm{ha}^{-1}$, para os experimentos 1 e 2, respectivamente); $\mathrm{N}_{\mathrm{c}}$ é o nível crítico de $\mathrm{N}-\mathrm{N}-\mathrm{NO}_{3}$ na camada de 0 -20 cm do solo (52 kg ha-1), derivado dos resultados de Guimarães (1998); e E é a eficiência da utilização do $\mathrm{N}$ aplicado, considerando 50\% para o tomateiro (Sweeney et al., 1987).

As doses calculadas de $\mathrm{N}$ pelos critérios PESF, QECS e DRCO foram parceladas a cada 14 dias, sendo $10 \%$ aos 14 dias após o transplante (DAT) e 15\% aos 28; 42; 56; 70; 84 e 98 DAT. No critério QENF a quantidade do fertilizante nitrogenado (sulfato de amônio, $18 \%$ de N) foi parcelada, sendo 5; 10; 22; 26; 19; 10 e $8 \%$ aos 14; 28; 42; 56; 70; 84 e 98 DAT, respectivamente. No critério DRTR a quantidade do fertilizante nitrogenado foi aplicado totalmente no momento do transplante. Os tratamentos foram distribuídos no delineamento de blocos ao acaso, com três repetições no experimento 1 e quatro no experimento 2 .

Cinco dias antes do transplante das mudas foi realizada a adubação de plantio. No experimento 1 , foram aplicadas nos sulcos as misturas de $600 \mathrm{~kg} \mathrm{ha}^{-1}$ de $\mathrm{P}_{2} \mathrm{O}_{5}, 64 \mathrm{~kg} \mathrm{ha}^{-1}$ de $\mathrm{K}_{2} \mathrm{O}$, correspondente a $16 \%$ da dose total de $\mathrm{K}_{2} \mathrm{O}$, e $18 \mathrm{~kg}$ $\mathrm{ha}^{-1}$ de $\mathrm{Mg}$. No experimento 2, a mistura aplicada nos sulcos continha $24,5 \mathrm{~kg}$ de $\mathrm{K}_{2} \mathrm{O}$, correspondente a $16 \%$ da dose total de $\mathrm{K}_{2} \mathrm{O}$, e $18 \mathrm{~kg} \mathrm{ha}^{-1}$ de $\mathrm{Mg}$. As 
doses de fósforo e potássio foram determinadas de acordo com a análise química do solo e as recomendações para a cultura do tomate fertirrigado segundo Fontes (1999). Em ambos experimentos, o restante do $\mathrm{K}_{2} \mathrm{O}$ e $1 \mathrm{~kg} \mathrm{ha}^{-1}$ de $\mathrm{B}, 3$ $\mathrm{kg} \mathrm{ha}^{-1}$ de Zn e $78 \mathrm{~g} \mathrm{ha}^{-1}$ de Mo, nas formas de bórax, sulfato de zinco e molibdato de sódio, foram aplicados em cobertura, via água de irrigação, em seis parcelamentos aos 14; 28; 42; 56; 70 e 84 DAT.

As mudas foram transplantadas para o local definitivo quando alcançaram 10 a $15 \mathrm{~cm}$ de altura e apresentavam quatro a seis folhas. Imediatamente antes do transplante, foi instalado o sistema de fertirrigação, com gotejadores distanciados em $60 \mathrm{~cm}$ e dispostos sobre o solo paralelamente aos sulcos e linhas de transplante. As mudas foram transplantadas no espaçamento de $0,6 \mathrm{~m}$ entre plantas e 1,0 m entre fileiras. Cada parcela foi composta de quatro plantas úteis.

Foi utilizado o híbrido 'Carmen', longa vida, tipo salada e de crescimento indeterminado. O tomateiro foi conduzido com dois caules, com tutoramento vertical, em fitilhos de plástico, com cinco cachos no caule principal e quatro no caule secundário. Em cada cacho foi permitido o crescimento de seis frutos. As práticas culturais foram efetuadas de acordo com as recomendações de Fontes \& Silva (2002). As irrigações foram diárias e o volume de água aplicado foi calculado pelo método do lisímetro, instalado no interior do ambiente protegido.

Os frutos foram colhidos quando apresentavam a coloração vermelha, separados em sem e com defeitos, contados e classificados. Os frutos sem defeitos foram classificados como comerciais de acordo com seu diâmetro transversal, segundo norma do Ministério da Agricultura e Reforma Agrária (Portaria $n^{0} 553$ de 30.08.95, publicada no DOU de 19/09/95), considerando Gigante $\varnothing>100 \mathrm{~mm}$, Grande $80<\varnothing<100$ $\mathrm{mm}$, Médio $65<\varnothing<80 \mathrm{~mm}$ e Pequeno $50<\varnothing<65 \mathrm{~mm}$. Os frutos com defeito e aqueles com diâmetro transversal $<50$ mm foram classificados como não comerciais.

A produção total foi obtida pelo somatório da produção comercial e não- comercial. A produção ponderada de frutos foi calculada utilizando fatores de ponderação de 0,635 e 0,400 para transformar em classe de frutos grande as classes médio e pequeno, respectivamente.

Na quarta colheita, três frutos comercializáveis, escolhidos ao acaso, foram pesados e secos em estufa a $70^{\circ} \mathrm{C}$ até atingirem massa constante, sendo determinados os teores de matéria seca. As amostras de frutos secos foram moídas em moinho tipo Wiley, equipado com peneira de 20 mesh, e submetidas à extração em água para a determinação do N-NO $\mathrm{N}_{3}$, de acordo com Cataldo et al. (1975) e à digestão sulfúrica para determinação do N-orgânico ( $\mathrm{N}-\mathrm{NH}_{4}$ incorporado em compostos orgânicos), pelo método de Nessler (Jackson, 1958). Pelo somatório do teor de N-orgânico $\left(\mathrm{N}-\mathrm{NH}_{4}\right)$ e $\mathrm{N}-\mathrm{NO}_{3}$ foi determinada a quantidade de $\mathrm{N}$ total.

Após a última colheita de frutos, as plantas úteis de cada parcela foram cortadas rente ao solo para a determinação das massas das matérias secas e do teor de N-orgânico e do $\mathrm{N}-\mathrm{NO}_{3}$ de folhas e caule, de acordo com a metodologia descrita para os frutos. Para a determinação da quantidade de matéria seca total das plantas foi utilizada a soma das massas das matérias secas de folhas, caules e frutos. Pela multiplicação da quantidade de matéria seca pelo teor de $\mathrm{N}$ total na matéria seca foi obtido o conteúdo de $\mathrm{N}$ na parte aérea do tomateiro.

Após a retirada das plantas, para a determinação do teor de $\mathrm{N}-\mathrm{NO}_{3}$ residual, amostras de solo foram obtidas a $20 \mathrm{~cm}$ de profundidade, em ponto distanciado $10 \mathrm{~cm}$ das plantas, sobre o sulco de transplante e em $10 \mathrm{~cm}$ entre sulco de transplante. Nestas foi extraído o $\mathrm{N}-\mathrm{NO}_{3}$ utilizando-se $\mathrm{KCl} 1 \mathrm{~mol} \mathrm{~L}^{-1}$, na relação solo:extrator de 1:10. O N-NO ${ }_{3}$ presente no extrato foi dosado por metodologia simplificada, baseada no método do salicilato proposta por Yang et al. (1998).

Para cada tratamento, os valores do conteúdo de $\mathrm{N}$ total na parte aérea e da quantidade de $\mathrm{N}-\mathrm{NO}_{3}$ residual no solo foram utilizados para o calculo do balanço de entrada $\left(N_{\mathrm{ENT}}\right)$ e saída $\left(N_{\mathrm{SAI}}\right)$ de
$\mathrm{N}$ do sistema (Errebhi et al., 1998): $N_{\text {ENT }}=N_{\text {ini }}+N_{\text {fer }}+N_{\text {irr }} \quad N_{\text {SAI }}=N_{\text {pla }}+N_{\text {fin }}$ Onde, $N_{\text {ini }}$ é o teor inicial de $\mathrm{N}-\mathrm{NO}_{3}$ na camada de $0-20 \mathrm{~cm}$ do solo $\left(\mathrm{kg} \mathrm{ha}^{-1}\right.$ de $\left.\mathrm{N}-\mathrm{NO}_{3}\right) ; N_{\text {fer }}$ é a dose do fertilizante nitrogenado ( $\mathrm{kg} \mathrm{ha}^{-1}$ de $\left.\mathrm{N}\right) ; N_{\text {irr }}$ é a quantidade de $\mathrm{N}-\mathrm{NO}_{3}$ proveniente da água de irrigação $\left(\mathrm{kg} \mathrm{ha}^{-1}\right.$ de $\left.\mathrm{N}-\mathrm{NO}_{3}\right) ; N_{\text {pla }}$ é o conteúdo de $\mathrm{N}$ na parte aérea das plantas (kg ha-1 de N); $N_{\text {fin }}$ é o teor de $\mathrm{N}-\mathrm{NO}_{3}$ presente na camada de $0-20 \mathrm{~cm}$ do solo, no final do período de cultivo (kg ha-1 de $\mathrm{N}-\mathrm{NO}_{3}$ ).

De acordo com a terminologia encontrada em Delogu et al. (1998) e LópezBellido \& López-Bellido (2001), ou adaptadas de Siddiqi \& Glass (1981) e Sowers et al. (1994), foi calculada a eficiência de uso do fertilizante nitrogenado, expressa por diferentes modos:

Eficiência do uso do N (EUSN) =

Produçāo de frutos $\mathrm{N}_{\mathrm{x}}+\mathrm{N}_{\text {oglo }}+\mathrm{N}_{\text {mia }}$

Eficiência de utilização do N $($ EUTN $)=\frac{\text { Produçāo de frutos }}{\text { QNPA }}$

Eficiência de absorção do N

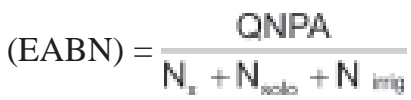

Eficiência agronômica do N $($ EAGN $)=\frac{\text { Produçāo } N_{\mathrm{x}}-\text { Pr oduçāo } N_{0}}{N_{x}}$

Eficiência de recuperação do fertilizante $($ ERFN $)=\frac{\text { QNPA N } N_{x}-\text { QNPA N }}{N_{v}} \times 100$

Nestas, $\mathrm{N}_{\mathrm{x}}=$ dose aplicada em cada tratamento $\left(\mathrm{kg} \mathrm{ha}^{-1}\right.$ de $\left.\mathrm{N}\right)$; $\mathrm{N}_{\text {solo }}=$ teor de $\mathrm{N}-\mathrm{NO}_{3}$ do solo $\left(\mathrm{kg} \mathrm{ha}^{-1}\right.$ de $\mathrm{N}-\mathrm{NO}_{3}$ ); $\mathrm{N}_{\text {irrig }}$ = teor de $\mathrm{N}_{-} \mathrm{NO}_{3}$ na água de irrigação ( $\mathrm{kg} \mathrm{ha}^{-1}$ de $\mathrm{N}-\mathrm{NO}_{3}$ ); Produção $\mathrm{N}_{\mathrm{x}}=$ produção total de frutos no tratamento x (kg $h^{-1}$ ); Produção $\mathrm{N}_{0}=$ produção total de frutos na testemunha ( $\mathrm{kg} \mathrm{ha}^{-1}$ ); QNPA $\mathrm{N}_{\mathrm{x}}$ = quantidade de $\mathrm{N}$ da parte aérea da planta no tratamento $\mathrm{x}\left(\mathrm{kg} \mathrm{ha}^{-1}\right.$ de $\left.\mathrm{N}\right)$; QNPA $\mathrm{N}_{0}=$ quantidade de $\mathrm{N}$ na parte aérea da planta na testemunha (kg ha-1 de N); QNPA = quantidade de $\mathrm{N}$ na parte aérea ( $\mathrm{kg} \mathrm{ha}^{-1}$ de $\left.\mathrm{N}\right)$.

Os dados obtidos foram submetidos à análise de variância e as médias dos tratamentos foram comparadas pelo teste de Tukey, a 5\% de probabilidade. 
Tabela 1. Produção total (PT), comercial (PC) e ponderada (PP) de frutos do tomateiro, em função dos tratamentos nos experimentos 1 e 2 (total yield (PT), commercial yield (PC) and ponderated yield of tomato fruits depending on treatments in experiments 1 and 2). Viçosa, UFV, 2003.

\begin{tabular}{|c|c|c|c|c|c|}
\hline \multirow{2}{*}{ Experimento } & \multirow{2}{*}{ Tratamento } & \multirow{2}{*}{$\begin{array}{c}\text { Dose de N } \\
\left(\mathrm{kg} \mathrm{ha}^{-1}\right)\end{array}$} & \multicolumn{3}{|c|}{ Produção (t ha-1) } \\
\hline & & & PT & PC & PP \\
\hline \multirow{6}{*}{1} & PESF & 538,8 & 106,6 a & $103,5 \mathrm{a}$ & $61,5 \mathrm{a}$ \\
\hline & QECS & 296,8 & $100,0 \mathrm{a}$ & 96,4 a & $54,6 a b$ \\
\hline & QENF & 439,2 & 102,8 a & $99,1 \mathrm{a}$ & $56,1 \mathrm{ab}$ \\
\hline & DRCO & 280,0 & 93,7 a & 91,5 a & $53,7 a b$ \\
\hline & DRTR & 280,0 & 104,5 a & $101,1 \mathrm{a}$ & 59,3 a \\
\hline & TEST & 0,0 & $68,3 \mathrm{~b}$ & $64,5 \mathrm{~b}$ & $37,5 \mathrm{~b}$ \\
\hline \multirow{6}{*}{2} & PESF & 570,8 & $103,1 \mathrm{a}$ & 98,0 a & 57,5 \\
\hline & QECS & 24,4 & $77,6 a b$ & $72,9 a b$ & 41,8 \\
\hline & QENF & 439,2 & $88,8 a b$ & $83,9 a b$ & 47,1 \\
\hline & DRCO & 280,0 & $94,3 a b$ & $89,8 \mathrm{ab}$ & 55,5 \\
\hline & DRTR & 280,0 & $101,7 a b$ & 98,1 a & 57,8 \\
\hline & TEST & 0,0 & 71,7 b & $68,2 \mathrm{~b}$ & 40,3 \\
\hline
\end{tabular}

Em cada experimento, médias seguidas pela mesma letra não diferem significativamente entre si na coluna a 5\% de probabilidade, pelo teste de Tukey. (means followed by the same letter in the column did not differ from each other, Tukey 5\%).

\section{RESULTADOS E DISCUSSÃO}

Em ambos experimentos as produções total e comercial de frutos não diferiram significativamente entre os tratamentos que receberam o $\mathrm{N}$ (Tabela 1 ). Maiores produções foram obtidas pela utilização do critério PESF, que foi baseado na produtividade esperada de frutos e na capacidade de fornecimento de $\mathrm{N}$ pelo solo e pelo fertilizante. Esse critério propiciou a aplicação de 538,8 e $570,8 \mathrm{~kg} \mathrm{ha}^{-1}$ de N, de forma parcelada a cada 14 dias, nos experimentos 1 e 2 , respectivamente. Como o critério PESF levava em consideração o teor de $\mathrm{N}^{-\mathrm{NO}_{3}}$ no solo para a determinação da quantidade de $\mathrm{N}$ a ser aplicado, o resultado obtido em relação à produção comprova o conceito que o conteúdo de $\mathrm{N}-\mathrm{NO}_{3}$ no solo pode ser utilizado para predizer a necessidade do fertilizante nitrogenado (Kerbs et al., 1973). Produtividade semelhante à do critério PESF foi obtida pela utilização do critério DRTR, que foi baseado na dose de $280 \mathrm{~kg} \mathrm{ha}^{-1}$ de N, recomendada por Fontes \& Guimarães (1999) e aplicada toda no momento do transplante. As produções obtidas com a utilização dos dois critérios foram acima de 96,7; 75,4 e 45,1 $\mathrm{t} \mathrm{ha}^{-1}$, obtidas com as doses de 241, 181 e $177 \mathrm{~kg} \mathrm{ha}^{-1}$ de $\mathrm{N}$, respectivamente, por Guimarães
(1998), no mesmo local de cultivo. Abdul-Baki et al. (1997) obtiveram produção comercial de $77,5 \mathrm{t} \mathrm{ha}^{-1}$ com dose de $\mathrm{N}$ de $314,7 \mathrm{~kg} \mathrm{ha}^{-1}$ sob condições de solo coberto com plástico (mulching).

O critério QECS, que foi baseado na quantidade esperada de $\mathrm{N}$ no fruto mais a capacidade de fornecimento de $\mathrm{N}$ pelo solo proporcionou alta produção de frutos e a recomendação de $296,8 \mathrm{~kg} \mathrm{ha}^{-1} \mathrm{de}$ $\mathrm{N}$ no experimento 1, próximo de $280 \mathrm{~kg}$ ha ${ }^{-1}$ de N recomendado por Fontes \& Guimarães (1999). Entretanto, no experimento 2, onde o solo apresentava teor de N$\mathrm{NO}_{3} 106,9 \%$ maior do que no experimenmendação de apenas $24,4 \mathrm{~kg} \mathrm{ha}^{-1}$ de N. Essa dose de $\mathrm{N}$ não foi suficiente para satisfazer a necessidade de $\mathrm{N}$ do tomateiro, resultando na redução na produção de frutos quando comparado aos demais critérios onde foi aplicado N. Dessa forma, o critério QECS, diferentemente do critério PESF, que também foi baseado no teor de $\mathrm{N}-\mathrm{NO}_{3}$ no solo, parece não ser preciso em determinar a quantidade de $\mathrm{N}$ a ser aplicada no tomateiro quando o teor inicial de $\mathrm{N}^{-} \mathrm{NO}_{3}$ no solo for elevado.

O critério QENF, que foi baseado na quantidade esperada de $\mathrm{N}$ nos frutos, portanto depende da estimativa da produção esperada de frutos, também apresentou resultados promissores para ser utilizado como critério para o manejo to 1, o critério QECS resultou na reco- da adubação nitrogenada. Entretanto, no experimento 1 houve grande perda de $\mathrm{N}$ do sistema solo-planta pela utilização desse critério (Tabela 2). Esses resultados confirmam aqueles de Scharf (2001), ao concluir que o critério baseado na quantidade esperada de $\mathrm{N}$ no fruto pode não ser adequado para a recomendação da dose de N, provavelmente por não levar em consideração as diferenças de quantidade e capacidade de fornecimento de $\mathrm{N}$ entre locais, ou seja, o conteúdo de $\mathrm{N}-\mathrm{NO}_{3}$ do solo.

As produtividades obtidas pela utilização dos critérios DRCO e DRTR indicam que, sob condições de ambiente protegido e irrigação por gotejo, o fertilizante nitrogenado pode ser aplicado preferencialmente no sulco de transplante, de forma mais fácil e menos trabalhosa, sem a necessidade de aplicações freqüentes em cobertura via água de irrigação. Rhoads et al. (1996) e Locascio et al. (1997) também observaram que a produção de frutos de tomate foi semelhante quando o fertilizante nitrogenado foi aplicado todo no momento do transplante ou em cobertura. Isso ocorreu, provavelmente, pela baixa perda de $\mathrm{N}$ via lixiviação e ausência de alta salinidade no solo, devido ao efeito da aplicação de água em quantidade adequada e constante, localizada próxima à região de maior concentração das raízes, por gotejamento, e, provavelmente, devido à aplicação parcelada do $\mathrm{K}$ que pode ter evitado a salinização momentânea do solo. Provavelmente, reduzida lixiviação pode ter ocorrido também, porque o solo da área apresentava altas capacidades de troca aniônica e de adsorção de nitrato por ter argila do tipo 1:1 e óxidos de ferro e alumínio (Singh \& Kanehiro, 1969). Solos com essas características retardam o movimento do íon em relação ao movimento de água, minimizando o efeito da lixiviação (Belinni et al., 1996), destacadamente em ambiente protegido, e aumentando a eficiência da adubação nitrogenada.

De forma geral, as eficiências de uso, utilização e agronômica do $\mathrm{N}$ diminuíram à medida que a quantidade de $\mathrm{N}$ aplicada aumentou nos dois experimentos (Tabela 2). Para Johnson \& Raun (2003), a diminuição na eficiência de 
Tabela 2. Balanço de entrada ( $\left.N_{\mathrm{ENT}}\right)$ e saída $\left(N_{\mathrm{SAI}}\right)$ de $\mathrm{N}\left(\mathrm{kg} \mathrm{ha}^{-1}\right)$, eficiência de uso (EUSN), de utilização (EUTN), agronômica (EAGN) e de recuperação do $\mathrm{N}$ (ERFN), em função dos tratamentos nos experimentos 1 e 2 (evaluation of $\mathrm{N}\left(\mathrm{kg} \mathrm{ha}^{-1}\right)$ entering $\left(N_{\mathrm{ENT}}\right)$ and expense $\left(N_{\mathrm{SAI}}\right)$ efficiency of use (EUSN), utilization (EUTN) agronomic (EAGN) and recovering of N (ERFN), resulting from the experiments 1 and 2). Viçosa, UFV, 2003.

\begin{tabular}{|c|c|c|c|c|c|c|c|c|c|c|c|c|}
\hline \multirow{2}{*}{ Experimento } & \multirow{2}{*}{ Tratamento } & \multicolumn{3}{|c|}{ Entrada } & \multicolumn{3}{|c|}{ Saída } & \multirow{2}{*}{$\mathrm{N}_{\mathrm{ENT}}-\mathrm{N}_{\mathrm{SAl}}{ }^{1}$} & \multirow{2}{*}{\multicolumn{2}{|c|}{$\frac{\text { EUTN }}{\left(\mathrm{kg} \mathrm{kg}^{-1}\right)}$}} & \multirow[t]{2}{*}{ EAGN } & \multirow{2}{*}{$\begin{array}{c}\text { ERFN } \\
(\%)\end{array}$} \\
\hline & & $\mathbf{N}_{\text {ini }}$ & $\mathbf{N}_{\text {fert }}+\mathbf{N}_{\text {irr }}$ & $\mathbf{N}_{\mathrm{ENT}}$ & $\mathbf{N}_{\text {pla }}$ & $\mathbf{N}_{\text {fin }}$ & $\mathbf{N}_{\mathrm{SAI}}$ & & & & & \\
\hline \multirow{6}{*}{1} & PESF & 123,2 & 541,9 & 665,1 & 282,1 & 298,8 & 580,9 & $84,5 \mathrm{~b}$ & $160,3 \mathrm{~b}$ & 380,5 & 71,1 & 24,0 \\
\hline & QECS & 123,2 & 299,9 & 423,1 & 214,4 & 220,1 & 434,5 & $-11,4 a b$ & $236,4 \mathrm{~b}$ & 467,7 & 107,0 & 20,9 \\
\hline & QENF & 123,2 & 442,3 & 565,5 & 250,9 & 191,0 & 441,9 & $123,6 \mathrm{a}$ & $181,7 \mathrm{~b}$ & 410,2 & 78,5 & 22,4 \\
\hline & DRCO & 123,2 & 283,1 & 406,3 & 205,4 & 200,1 & 405,5 & $0,8 a b$ & $230,6 \mathrm{~b}$ & 459,1 & 90,8 & 18,9 \\
\hline & DRTR & 123,2 & 283,1 & 406,3 & 228,0 & 476,9 & 704,9 & $-298,6$ c & $257,1 \mathrm{~b}$ & 458,4 & 129,1 & 27,0 \\
\hline & TEST & 123,2 & 3,1 & 126,3 & 152,3 & 58,5 & 210,8 & $-84,5 a b c$ & 540,7 a & 460,7 & - & - \\
\hline \multirow{6}{*}{2} & PESF & 101,1 & 573,9 & 675,0 & 258,8 & 803,5 & 1062,3 & $-387,3 a b$ & $156,6 \mathrm{~b}$ & 400,3 & 55,1 & 19,6 \\
\hline & QECS & 259,4 & 27,5 & 286,9 & 150,6 & 168,3 & 318,9 & $-32,0$ a & $313,9 \mathrm{~b}$ & 521,9 & 243,7 & 13,1 \\
\hline & QENF & 473,6 & 442,3 & 915,9 & 213,7 & 805,9 & 1019,6 & $-103,7 a b$ & $98,9 \mathrm{~b}$ & 417,1 & 39,1 & 15,2 \\
\hline & DRCO & 151,8 & 283,1 & 434,9 & 222,3 & 575,4 & 797,7 & $-362,8 a b$ & $219,8 \mathrm{~b}$ & 425,8 & 80,9 & 26,8 \\
\hline & DRTR & 85,6 & 283,1 & 368,7 & 225,1 & 662,0 & 887,1 & $-518,4$ b & $276,1 \mathrm{~b}$ & 455,8 & 107,1 & 27,9 \\
\hline & TEST & 29,8 & 3,1 & 32,9 & 147,3 & 140,5 & 287,8 & $-254,9 a b$ & $2269,3 \mathrm{a}$ & 489,6 & - & - \\
\hline
\end{tabular}

${ }^{1}$ Valores negativos e positivos correspondem à quantidade provável de $\mathrm{N}$ que foi mineralizado e perdido, respectivamente; Em cada experimento, médias seguidas pela mesma letra não diferem significativamente entre si na coluna a 5\% de probabilidade, pelo teste de Tukey (negative and positive values correspond to the probable mineralized and lost $\mathrm{N}$, respectively; In each experiment, means followed by the same letter in the colums did not differ from each other, Tukey 5\%).

uso é o resultado da perda de $\mathrm{N}$ do sistema solo-planta e esta perda ocorre em proporção direta ao grau em que o $\mathrm{N}$ presente no solo está em excesso. Portanto, os critérios com as maiores doses de $\mathrm{N}$ aplicadas resultaram em excesso de $\mathrm{N}$ no solo e, conseqüentemente, menor eficiência de uso e de absorção do $\mathrm{N}$ foi observada. Redução na eficiência do $\mathrm{N}$ disponível com o aumento na dose de $\mathrm{N}$ indica que maior proporção de $\mathrm{N}$ fornecido não foi recuperado nos tecidos da planta ou ficou retido no perfil do solo à profundidade do sistema radicular ou foi perdido para camadas mais profundas do solo (Sowers et al., 1994). Pela análise do balanço de entrada e saída, onde os critérios PESF e QENF apresentaram provável perda de $\mathrm{N}$ do sistema solo planta, pode-se hipotetizar que houve perda de $\mathrm{N}$ via lixiviação.

A eficiência de utilização do $\mathrm{N}$, que representa a habilidade da planta para transformar o $\mathrm{N}$ absorvido em produção (Delogu et al., 1998), não foi influenciada significativamente pelos critérios utilizados, em ambos experimentos, indicando que cada quilograma de $\mathrm{N}$ acumulado na parte aérea correspondeu à produção de 380,5 a 521,9 kg de frutos. Com tais valores é possível determinar que são necessários
1,92 a 2,63 kg de N para a produção de 1 t de frutos. Esses valores estão acima

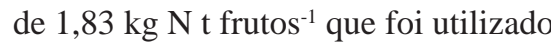
nos critérios QECS e QENF, e determinado com base nos resultados obtidos por Fayad (1998). Para Carvalho et al. (2004), a quantidade de $\mathrm{N}$ necessária para a produção de $1 \mathrm{t}$ de frutos pode variar de 2,1 a 3,8 kg de N. Provavelmente, a utilização de valor mais baixo para a produção de $1 \mathrm{t}$ de frutos contribuiu para que as doses de $\mathrm{N}$ determinadas pela utilização dos critérios QECS e QENF não tenham propiciado as maiores produções de frutos (Tabela 1) e eficiências de uso e de recuperação do $\mathrm{N}$ derivado do fertilizante (Tabela 2).

Apesar de não ter sido encontrada diferença significativa entre os critérios estudados para a eficiência de recuperação do $\mathrm{N}$ do fertilizante, em ambos experimentos, pode ser observado que maior proporção do $\mathrm{N}$ proveniente do fertilizante foi utilizada quando o $\mathrm{N}$ foi aplicado totalmente no momento do transplante, em quantidade recomendada para a cultura do tomateiro (Tabela 2). Independentemente da quantidade de $\mathrm{N}$ utilizada nos diferentes critérios, a eficiência de recuperação de $\mathrm{N}$ foi baixa, variando de 13,1 a 27\%. Hills et al. (1983) relataram que a eficiência de recuperação do $\mathrm{N}$ derivado do fertilizan- te, na forma de sulfato de amônio, foi de $27 \%$ para o tomateiro. Sainju et al. (1999) observaram eficiência de recuperação do fertilizante nitrogenado variável entre experimentos e entre doses de $\mathrm{N}$ aplicadas, com valores de 2 a 28,8\%. Esses valores estão abaixo daqueles obtidos por Sweeney et al. (1987) e Scholberg et al. (2000) ao observarem eficiências de recuperação do $\mathrm{N}$ pelo tomateiro variando de 31,5 a 53\% quando foi aplicado 224 $\mathrm{kg} \mathrm{ha}^{-1}$ de $\mathrm{N}$ e de 36 a 74\%, dependendo da dose de $\mathrm{N}$ aplicada.

A baixa eficiência de recuperação do $\mathrm{N}$ pelo tomateiro indica que grande quantidade de $\mathrm{N}$ permaneceu no solo no final do ciclo do tomateiro, conforme observado nos valores de $\mathrm{N}_{\text {fin }}$ (Tabela 2), que corresponde ao teor de $\mathrm{N}-\mathrm{NO}_{3}$ residual presente na camada de $0-20 \mathrm{~cm}$ do solo, no final do período de cultivo. Isso pode não ser desejável, pois a adubação nitrogenada tem que ser conduzida de tal maneira que máxima produtividade seja obtida com menor teor de $\mathrm{N}-\mathrm{NO}_{3}$ possível no solo, no final do período de cultivo (Ibrikci et al., 2001), reduzindo o potencial de perda desse íon via lixiviação ou desnitrificação.

Os critérios PESF e DRTR proporcionaram maior produtividade e eficiência na recuperação do $\mathrm{N}$ derivado do 
fertilizante. A utilização do primeiro depende da facilidade de determinações do teor de $\mathrm{N}_{-} \mathrm{NO}_{3}$ no solo e da capacidade de fornecimento de $\mathrm{N}$ pelo fertilizante para a produção de 1 tonelada de frutos. A utilização do critério DRTR depende da disponibilidade de recomendação de dose de $\mathrm{N}$ baseada em resultados de experimentos realizados em anos anteriores.

\section{AGRADECIMENTOS}

Somos gratos à FAPEMIG que propiciou parte do recursos financeiros para a execução do trabalho e ao CNPq pelas Bolsas de Pós-Graduação e de Pesquisa concedidas aos autores.

\section{REFERÊNCIAS}

ABDUL-BAKI AA; TEASDALE JR; KORCAK RF. 1997. Nitrogen requirements of freshmarket tomatoes on hairy vetch and black polyethylene mulch. HortScience 32: 217-221.

BELINNI G; SUMNER ME; RADICLIFFE DE; QAFOKU NP. 1996. Anion transport through of highly weathered acid soil: adsorption and retardation. Soil Science Society of American Journal 60: 132-137.

CARVALHO JG; BASTOS ARR; ALVARENGA MAR. 2004. Nutrição mineral e adubação. In: ALVARENGAMAR (Ed.). Tomate: produção em campo, em casa-de-vegetação e em hidroponia. Lavras: Editora UFLA, p.61-120.

CATALDO DA; HARRON M; SCHRADER LE; YOUNES VL. 1975. Rapid colorimetric determination of nitrate in plant tissue by nitration of salicylic acid. Communication in Soil Science and Plant Analysis 6: 71-80.

DELOGU G; CATTIVELLI L; PECCHIONI N; FALCIS D; MAGGIORE T; STANCA AM. 1998. Uptake and agronomic efficiency of nitrogen in winter barley and winter wheat. European Journal of Agronomy 9: 11-20.

ERREBHI M; ROSEN CJ; GUPTA SC; BIRONG DE. 1998. Potato yield response and nitrate leaching as influenced by nitrogen management. Agronomy Journal 90: 10-15.

FAYAD JA. 1998. Absorção de nutrientes, crescimento e produção do tomateiro cultivado em condições de campo e de estufa. Viçosa: UFV, $81 \mathrm{f}$. (Tese mestrado) - UFV, Viçosa.

FONTES PCR. 1999. Sugestões de adubações para hortaliças. In: RIBEIRO AC; GUIMARÃES PTG; ALVAREZ VVH (Ed.). 1999. Comissão de fertilidade do solo do Estado de Mi- nas Gerais. Recomendações para uso de corretivos e fertilizantes em Minas Gerais. $5^{a}$ aproximação. Viçosa: Imprensa Universitária, p.171-174

FONTES PCR; FAYAD JA; GRAÇA RN. 2004. Utilização de característica agronômica como critério de controle das doses de nitrogênio e de potássio para a adubação do tomateiro. Horticultura Brasileira 22: 489, suplemento 2004.

FONTES PCR; GUIMARÃES TG. 1999. Manejo dos fertilizantes nas culturas de hortaliças cultivadas em solo, em ambiente protegido. Informe Agropecuário 20: 36-44.

FONTES PCR; SILVA DJH. 2002. Produção de tomate de mesa. Viçosa: Aprenda Fácil, 195 p.

GUIMARÃES TG. 1998. Nitrogênio no solo e na planta, teor de clorofila e produção do tomateiro, no campo e na estufa, influenciados por doses de nitrogênio. Viçosa: UFV, 184 f. (Tese doutorado) - UFV, Viçosa.

HECKMAN JR. 2002. In-season soil nitrate testing as a guide to nitrogen management for annual crops. HorTechnology 12: 706-710.

HILLS FJ; BROADBENT FE; LORENZ OA. 1983. Fertilizer nitrogen utilization by corn, tomatoes and sugarbeets. Agronomy Journal 75: 423-426.

IBRIKCI H; BUYUK G; YAGBASANLAR T; KEKLIKCI Z; TKLU F; GUZEL N; OZKAN H. 2001. Contribution of soil mineral nitrogen in wheat production. Journal of Plant Nutrition 24: 1871-1883.

JACKSON ML. 1958. Soil chemical analysis. Englewood cliffs: Prentice Hall, 458 p.

JOHNSON GV; RAUN WR. 2003. Nitrogen response index as a guide to fertilizer management. Journal of Plant Nutrition 26: 249-262.

KERBS LD; JONES JP; THIESSEN WL; PARKS FP. 1973. Correlation of soil test nitrogen with potato yield. Communications in Soil Science and Plant Analysis 4: 269-278.

LOCASCIO SJ; HOCHMUTH GJ; RHOADS FM; OLSON SM; SMAJSTRLA AG; HANLON EA. 1997. Nitrogen and potassium application scheduling effects on drip-irrigated tomato yield and leaf tissue analysis. HortScience 32: 230-235.

LÓPEZ-BELLIDO RJ; LÓPEZ-BELLIDO L. 2001. Efficiency of nitrogen in wheat under Mediterranean conditions: effect of tillage, crop rotation and $\mathrm{N}$ fertilization. Field Crops Research 71: 31-46.

NEETESON JJ. 1995. Nitrogen management for intensively grown arable crops and field vegetables. In: BACON PE (Ed.). Nitrogen fertilization in the environment. New York:
Marcel Dekker, p.295-325.

RHOADS FM; OLSON SM; HOCHMUTH GJ; HNALON EA. 1996. Yield an petiole-sap nitrate levels of tomato with $\mathrm{N}$ rates applied preplant or fertigated. Soil Crop Sciences Society of Florida Proceedings 55: 20-22.

SAINJU UM; SINGH BP; RAHMAN S; REDDY VR. 1999. Soil nitrate-nitrogen under tomato following tillage, cover cropping, and nitrogen fertilization. Journal Environment Quality 28: 1837-1844.

SCHARF P. 2001. Soil and plant tests to predict optimum nitrogen rates for corn. Journal of Plant Nutrition 24: 805-826.

SCHOLBERG J; MACNEAL BL; BOOTE KJ; JONES JW; LOCASCIO SJ; OLSON SM. 2000. Nitrogen stress effects on growth and nitrogen accumulation by filed-growth tomato. Agronomy Journal 92: 159-167.

SCHRÖDER JJ; NEETESON JJ; OENEMA O; STRUIK PC. 2000. Does crop or the soil indicate how to save nitrogen in maize production? Reviewing the state of the art. Field Crops Research 66: 151-164.

SIDDIQI MY; GLASS ADM. 1981. Utilization index: a modified approach to the estimation and comparison of nutrient utilization efficiency in plants. Journal of Plant Nutrition 4: 289-302.

SINGHI BR; KANEHIRO Y. 1969. Adsorption of nitrate in amorphous and koalinitic Hawaiian soils. Soil Science Society of American Proceedings 33: 681-683.

SOWERS KE; PAN WL; MILLER BC; SMITH JL. 1994. Nitrogen use efficiency of split nitrogen application in soft white winter wheat. Agronomy Journal 86: 942-948.

SWEENEY DW; GRAETZ DA; BOTTCHER AB; LOCASCIO SJ; CAMPBELL KL. 1987. Tomato yield and nitrogen recovery as influenced by irrigation method, nitrogen source, and mulch. HortScience 22: 27-29.

WADMAN WP; NEETESON JJ; ZWETSLOOT HJC. 1990. Development of nitrogen fertilizer recommendations for potatoes and sugar beet on the basis of soil testing. Kali-briefe (büntehof) 20: 171-180.

WARNCKE DD. 1996. Soil and plant tissue testing for nitrogen management in carrots. Communications in Soil Science and Plant Analysis 27: 597-605.

YANG JE; SKOGLEY EO; SCHAFF BE; KIM JJ. 1998. A simple spectrophotometric determination of nitrate in water, resin and soil extracts. Soil Science Society of American Journal 62: 1108-1115. 